\title{
Simulation of proton driven plasma wakefield acceleration
}

\author{
K. V. Lotov \\ Budker Institute of Nuclear Physics, 630090, Novosibirsk, Russia \\ Novosibirsk State University, 630090, Novosibirsk, Russia
}

(Received 28 October 2009; published 1 April 2010)

\begin{abstract}
Proton beam driven plasma wakefield acceleration was recently proposed as a way to bring electrons to $\mathrm{TeV}$ energy range in a single plasma section. Here we present a detailed numerical analysis of this acceleration scheme. We identify the main effects limiting acceleration efficiency and ultimate energy gain, and formulate optimum conditions for acceleration.
\end{abstract}

DOI: 10.1103/PhysRevSTAB.13.041301

PACS numbers: 41.75.Lx, 52.40.Mj

\section{INTRODUCTION}

Acceleration of particles in plasmas (or plasma wakefield acceleration) has now been studied as a possible way to high particle energies in laboratory experiments [1-4]. The plasma is an ionized medium that is difficult to destroy further, so it can support electric fields several orders of magnitude higher than those in traditional (metal) accelerating structures. The field in the plasma can be excited either by an intense laser pulse [5], or by a charged particle beam [6]. Both methods are experimentally proven to yield accelerating gradients of the order of $\mathrm{GeV} / \mathrm{cm}$ and energy gains beyond $1 \mathrm{GeV}[7,8]$.

The electron beam driven acceleration that currently holds the record of the total energy gain in a plasma [8] is subject to the so-called transformer ratio limit [9]. For realistic beam shapes, the accelerated (witness) beam sees roughly the same field amplitude as the drive beam (driver) does. Consequently, the energy gain of the witness cannot be much higher than the initial energy of driver particles. To accelerate electrons to $1 \mathrm{TeV}$, a $500 \mathrm{GeV}$ electron driver is necessary (that is unavailable), or several acceleration stages are to be made with several lower energy drivers (which is technically challenging [10]).

It was recently proposed to drive the wakefield by a proton beam rather than by an electron or a positron one [11]. Though the proton driven plasma wakefield acceleration (PDPWFA) is not free from the transformer ratio limit, it opens the way to $\mathrm{TeV}$-range electron energies due to availability of high-energy proton beams.

The protons are positively charged and have a greater mass than electrons. These two features make the physics of PDPWFA different from the well understood physics of electron-driven wakefield acceleration. A lower relativistic factor of the driver results in wave phase slippage and driver lengthening. The positive charge causes the plasma to operate in the "flow-in" nonlinear regime [12] rather than in the energetically efficient "blow-out" regime $[13,14]$. In this paper we analyze these effects and expound details of the numerical search that resulted in acceleration capabilities presented in [11]. We consecutively consider the necessity of an external quadrupole focusing (Sec. II), the optimum choice of the plasma density (Sec. III), the driver dynamics (Sec. IV), the optimum conditions for acceleration (Sec. V), and the consequences of deviations from these conditions (Sec. VI). As a reference point, we take driver and witness parameters from [11] (Table I). All simulations are made with two-dimensional quasistatic code LCODE $[15,16]$. Unless stated otherwise, we take the initial driver density of the form

$$
\begin{aligned}
& n_{P}=\frac{N_{P}}{2 \sigma_{r}^{2} \sigma_{z}(2 \pi)^{3 / 2}} e^{-r^{2} / 2 \sigma_{r}^{2}}\left[1+\cos \left(\sqrt{\frac{\pi}{2}} \frac{z}{\sigma_{z}}\right)\right], \\
& |z|<\sigma_{z} \sqrt{2 \pi} .
\end{aligned}
$$

The cosine distribution (1) is close to the Gaussian one, but is more convenient for simulations, since the beam density smoothly vanishes outside the interval of a finite length.

\section{TRANSVERSE EQUILIBRIUM OF THE DRIVER HEAD}

Plasma electrons need some time to respond to the driver push and create the wakefield. Therefore, the driver head sees no plasma focusing and propagates as in the plasma-

TABLE I. Basic set of beam and plasma parameters. Marked by asterisks are the values obtained from calculations (see the text).

\begin{tabular}{lcc}
\hline \hline Parameter & Symbol & Value \\
\hline Initial proton beam: & & \\
Population & $N_{P}$ & $10^{11}$ \\
Energy & $W_{P}$ & $1 \mathrm{TeV}$ \\
Rms energy spread & $\delta W_{P} / W_{P}$ & 0.1 \\
Longitudinal spread & $\sigma_{z}$ & $0.1 \mathrm{~mm}$ \\
Rms emittance & $\epsilon_{P}$ & $0.01 \mathrm{~mm} \mathrm{mrad}$ \\
Radius* & $\sigma_{r}$ & $0.42 \mathrm{~mm}$ \\
Electrons in witness bunch & $N_{e}$ & $1.5 \times 10^{10}$ \\
Initial electron energy & $W_{e 0}$ & $10 \mathrm{GeV}$ \\
Magnetic field gradient & $S$ & $0.5 \mathrm{~T} / \mathrm{mm}$ \\
Magnetic field period* & $L_{q}$ & $3 \mathrm{~m}$ \\
Average plasma density* & $n_{0}$ & $10^{15} \mathrm{~cm}^{-3}$ \\
\hline \hline
\end{tabular}


free space. Because of the initial angular spread, the driver head expands unless special care is taken to focus it. The importance of this effect can be seen from the following estimate.

Assume the typical amplitude of longitudinal fields is $\alpha$ times the wave breaking limit

$$
E_{0}=\frac{m c \omega_{p}}{e} \approx 0.96 \mathrm{GV} / \mathrm{m} \sqrt{\frac{n_{0}}{10^{14} \mathrm{~cm}^{-3}}},
$$

where $n_{0}$ is the plasma density, $\omega_{p}=\sqrt{4 \pi n_{0} e^{2} / m}$ is the plasma frequency, $m$ is the electron mass, $e$ is the elementary charge, and $c$ is the light velocity. The field $\alpha E_{0}$ will stop a particle of the energy $W_{P}$ and mass $m_{P}$ at the distance

$$
L_{0}=\frac{W_{P}}{e \alpha E_{0}}=\frac{\gamma_{0} m_{P} c}{m \omega_{p} \alpha},
$$

where $\gamma_{0}$ is the initial relativistic factor. To create the wakefield efficiently, the driver must be focused to the transverse size $\sigma_{r} \sim c / \omega_{p}$, thus having the equilibrium angular spread $\Delta \varphi=\epsilon_{P} / \sigma_{r} \sim \epsilon_{P} \omega_{p} / c$. If propagating freely for the distance (3), particles will shift transversely by the distance $\sim L_{0} \Delta \varphi$. We can neglect the angular spread, if this shift is small compared to the driver width $\sigma_{r}$, or if

$$
\frac{\epsilon_{P} \gamma_{0} m_{P}}{m \alpha} \ll \frac{c}{\omega_{p}} .
$$

In other words, the normalized emittance of the driver multiplied by the mass ratio determines the maximum plasma density for which the angular spread can be neglected. For available high-energy proton beams, the lefthand side of (4) is about a few centimeters even for the fullamplitude plasma wave $(\alpha \sim 1)$, which corresponds to extremely low plasma densities, below $10^{12} \mathrm{~cm}^{-3}$. An external focusing is thus necessary to prevent the proton driver from expansion.

The required focusing can be provided by external quadrupole magnets. As we show later, extremely strong field gradients are required to keep the driver within a reasonable size. For the same reason, the quadrupoles must cover the whole plasma section with a little space between them.

The quadrupoles makes the beam-plasma interaction essentially three dimensional. However, it is possible to model the effect of quadrupoles within the axisymmetric two-dimensional geometry by periodic radial pushes of varying sign given to beam particles [10]. In this case, we can calculate the average focusing force similarly to the ponderomotive one. Let $S$ be the maximum magnetic field gradient: $B=S r$. The radial force exerted on a relativistic proton is $F_{r}=e B$, and it oscillates with the time period $\tau_{q}=2 \pi / \omega_{q}=L_{q} / c$, where $L_{q}$ is the space period of the quadrupoles. For the sinelike varying field gradient, the time-average force is

$$
F_{q}=-\frac{c^{2}}{4 W_{P} \omega_{q}^{2}} \frac{d F_{r}^{2}}{d r}=-\frac{S^{2} L_{q}^{2} e^{2} r}{8 \pi^{2} W_{P}} .
$$

For the piecewise-constant focusing (half-period $S$, halfperiod $-S$ ), the force is $\kappa$ times greater, with

$$
\kappa=\frac{4}{\pi}\left(1+\frac{1}{3^{4}}+\frac{1}{5^{4}}+\cdots\right) \approx 1.29 .
$$

This series appears from the harmonic expansion of the rectangular pulse:

$$
\sum_{k=1,3,5, \ldots} \frac{2(-1)^{(k-1) / 2} \cos k t}{k \sqrt{\pi}}= \begin{cases}1, & t \in(-\pi / 2, \pi / 2), \\ -1, & t \in(\pi / 2,3 \pi / 2)\end{cases}
$$

the fourth power in contributions of higher harmonics appears since the magnetic force is squared and divided by $\omega_{q}^{2}$. The linear average focusing corresponds to the betatron function

$$
\beta=\sqrt{\frac{r W_{P}}{F_{q}}}=\frac{2 \pi \sqrt{2} W_{P}}{S L_{q} e \sqrt{\kappa}},
$$

whence we find the equilibrium radius of the beam head:

$$
\sigma_{r}=\sqrt{\epsilon_{P} \beta} .
$$

As we see, the longer the quadrupoles the stronger the focusing and the tighter the beam head. However, the length $L_{q}$ is limited by the requirement of small highfrequency radial oscillations that have the amplitude

$$
\delta r \approx \frac{e B c^{2}}{W_{P} \omega_{q}^{2}} .
$$

The amplitude is small $(\delta r \ll r)$, if

$$
L_{q} \ll 2 \pi \sqrt{\frac{W_{P}}{e S}} .
$$

We put here the initial proton energy since the driver head undergoes a little change in energy. The transverse dynamics of driver body and witness is determined by the much stronger plasma focusing and is free from this limitation.

For the state of the art field gradients of $0.5 \mathrm{~T} / \mathrm{mm}$ [17], the right-hand side of (9) is about $16 \mathrm{~m}$. The condition (9) must be fulfilled with some safety margin to avoid coherent pulsations of the driver head radius which result in pulsations of wakefield amplitude and phase. Thus for simulations we choose $S=0.5 \mathrm{~T} / \mathrm{mm}, L_{q}=3 \mathrm{~m}$ and find $\sigma_{r}=0.42 \mathrm{~mm}, \Delta \varphi=2.4 \times 10^{-5}$.

\section{PLASMA DENSITY}

For the linearly responding plasma, the optimum plasma density is determined by the driver length from the condition [18] 

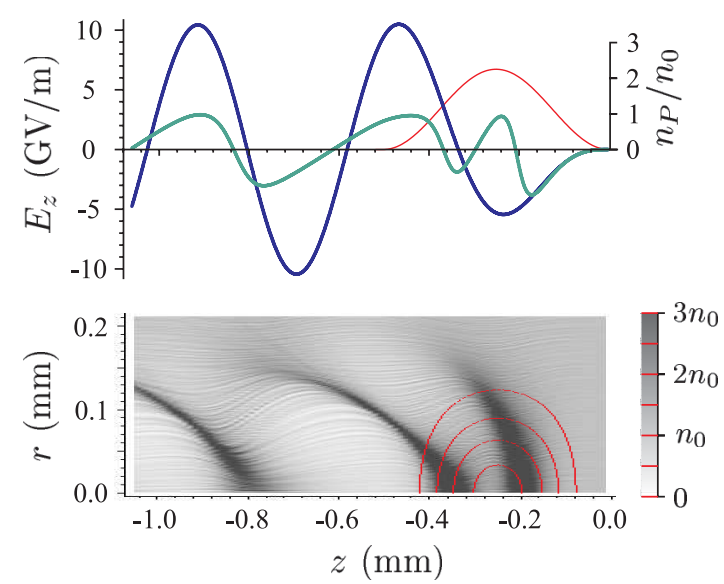

FIG. 1. (Color) Wakefield of the Gaussian beam for the "linear optimum" plasma density of $5.6 \times 10^{15} \mathrm{~cm}^{-3}$. On-axis electric field (top): actual (green) and predicted by the linear theory (blue); thin red line shows the on-axis driver density. Density of plasma electrons (bottom); red lines show the driver density.

$$
c / \omega_{p}=\sigma_{z} / \sqrt{2}
$$

which corresponds to $n_{0}=5.6 \times 10^{15} \mathrm{~cm}^{-3}$. As the plasma nonlinearity comes into play, the optimum shifts to lower plasma densities due to the "flow-in" effect. The driver attracts plasma electrons to the near-axis region, increases the local plasma frequency there, and decreases the local wakefield period (Fig. 1). The condition (10) thus refers to some averaged electron density near the driver rather than to the unperturbed plasma density.

To characterize the effect of plasma nonlinearity quantitatively, we plot in Fig. 2(a) the dependence of the maximum on-axis electric field $E_{z, \max }$ on the plasma density for three fixed-shape (not evolving) drivers with parameters listed in Table II. The driver radius in all variants is $\sigma_{r}=$ $c / \omega_{p}$ to approximate the width of a real driver that is in equilibrium with the focusing force. As we see, the field maximum is indeed shifted to lower densities.

The accelerating ability of the wakefield is determined not only by the field amplitude, but also by the transverse structure of the wave. The latter can be characterized by the energy stored per unit length or, equivalently, by the stopping power of the driver

$$
P_{\text {stop }}=-c \int e n_{P} E_{z} d \mathbf{r},
$$

which is shown in Fig. 2(b). The ratio $P_{\text {stop }} / E_{z, \max }$ [Fig. 2(c)] is roughly proportional to the number of electrons that can be accelerated by the wave. We see that this number and the field amplitude are simultaneously high for plasma densities about $10 \%-15 \%$ of the "linear optimum" value.

A comparison of the variants shows the effect of the driver length on wakefield properties. A longitudinal compression of the driver (variant 2 versus 1) results in a shift
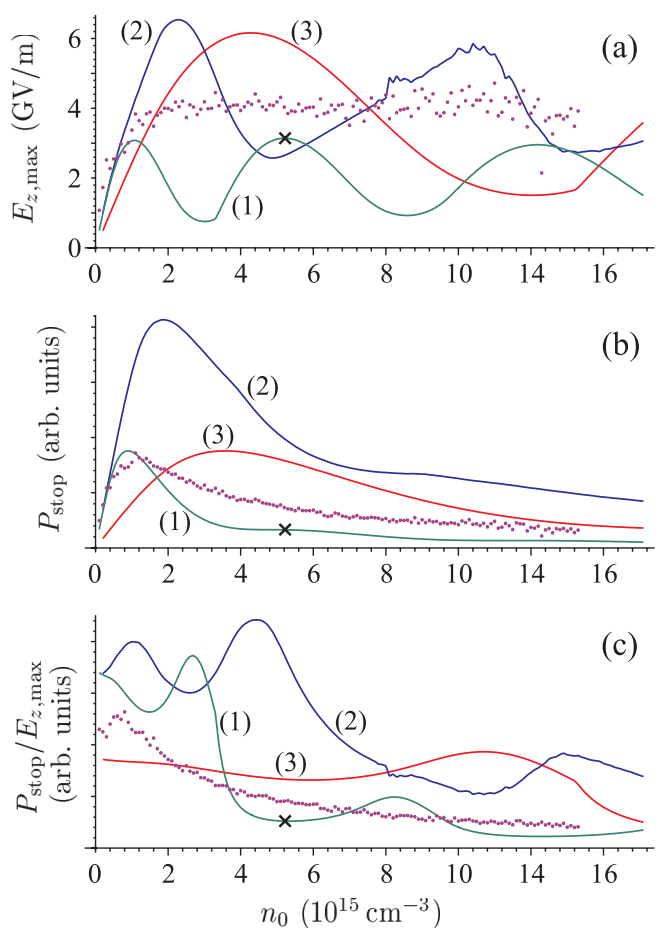

FIG. 2. (Color) Density dependence of the maximum on-axis electric field (a), stopping power of the driver (b), and ratio of the two (c) for fixed-shape beams (solid lines) and for the equilibrium beam (dots). The crosses show the "linear optimum" plasma density.

of the optimum plasma density to higher values and to approximately proportional increase of both field amplitude and stopping power; number of accelerated electrons remains the same. A longitudinal cutting of the beam (variant 3 versus 1) also increases the accelerating field, but does not increase the stopping power, so that the number of accelerated electrons is proportionally smaller. Thus, the peak accelerating field is controlled mainly by the driver length, while the ratio of witness-to-driver populations is roughly constant. Note that these statements are approximate ones and may not be correct if we deviate too far from driver parameters listed in Table I.

The Gaussian-like driver shape (1) with the Gaussian distribution over transverse momenta is not an equilibrium driver state. Therefore, at the first meters of propagation, the driver evolves toward some transverse equilibrium, after which the wakefield changes slowly. It is this equilibrium wakefield that is to be optimized by the choice of the plasma density. Wave characteristics measured at $15 \mathrm{~m}$

TABLE II. Parameters of fixed-shape drivers.

\begin{tabular}{lccc}
\hline \hline Number & \multicolumn{2}{c}{ Parameters } & Color \\
\hline$(1)$ & $N_{P}=10^{11}$ & $\sigma_{z}=100 \mu \mathrm{m}$ & Green \\
$(2)$ & $N_{P}=10^{11}$ & $\sigma_{z}=50 \mu \mathrm{m}$ & Blue \\
$(3)$ & $N_{P}=5 \times 10^{10}$ & $\sigma_{z}=50 \mu \mathrm{m}$ & Red \\
\hline \hline
\end{tabular}


from the entrance are shown in Fig. 2 by dots. The equilibrium field is higher than that for the fixed-shape driver of the same population and length (variant 1), though the stopping power is the same. This is due to effective longitudinal cutting: head and tail of the beam are not pinched by the plasma, so the equilibrium driver is shorter that the initially injected beam.

As follows from Fig. 2, the optimum plasma density for the equilibrium beam with initial characteristics listed in Table I lies in the vicinity of $10^{15} \mathrm{~cm}^{-3}$, where the stopping power is maximal. In what follows we take this value as the reference one. The corresponding plasma skin depth $c / \omega_{p} \approx 0.168 \mathrm{~mm}$.

\section{EVOLUTION OF THE DRIVER}

Figure 3 shows the evolution of the proton beam as it propagates through the plasma. The coordinate $z$ is measured from the point moving with the speed of light. Shortly after entering the plasma, the beam comes to the equilibrium with the strong focusing force of the wave (Fig. 4). The angular spread blows up [Fig. 3(b)] as does the transverse emittance of the beam. The equilibrium beam shape is essentially non-Gaussian and strongly peaked near the axis [Fig. 3(a)]. Beam head and tail experience weak focusing and are not pinched by the wave, so the fresh equilibrium beam $(L=90 \mathrm{~m})$ is effectively shorter than the initially injected one $(L=0 \mathrm{~m})$. (a)
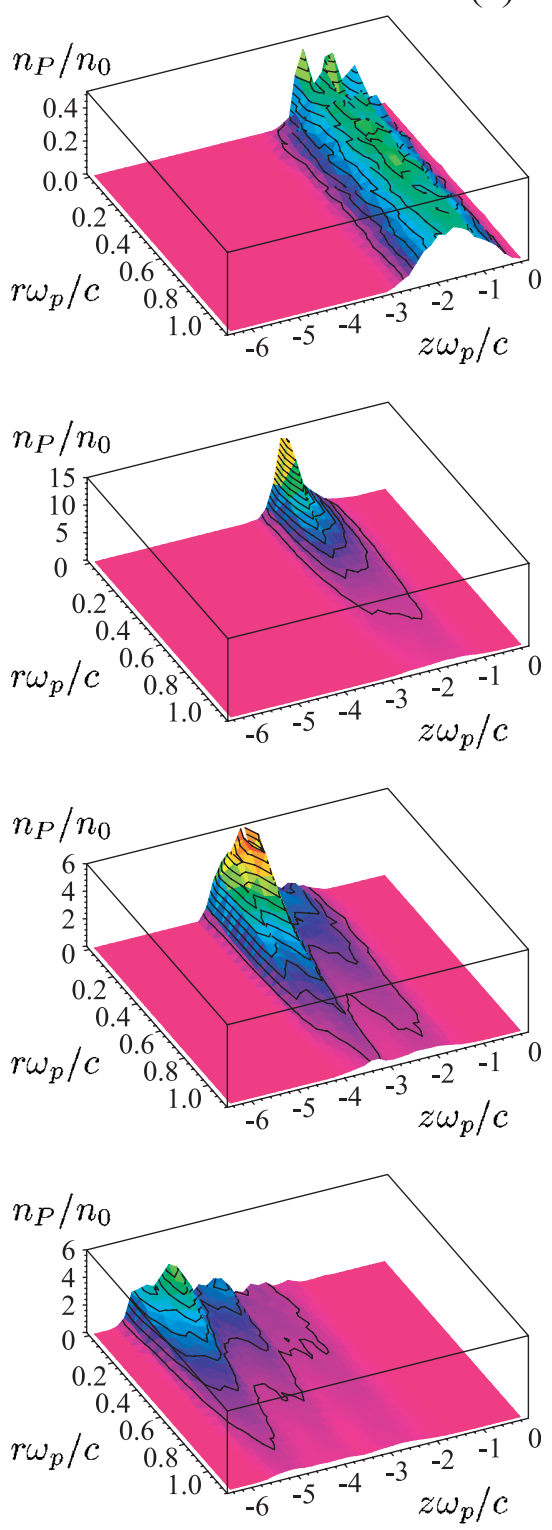

(b)
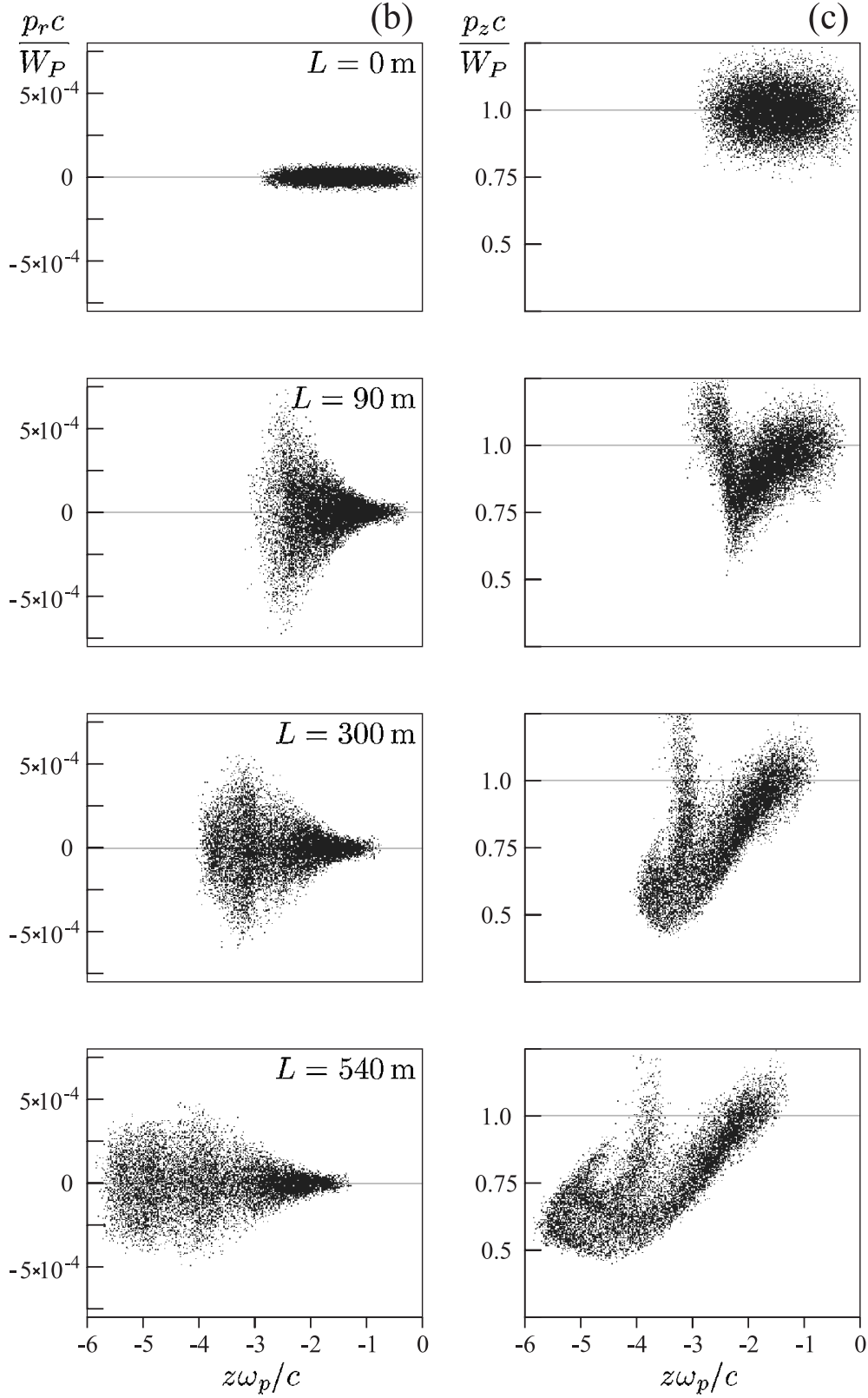

(c)
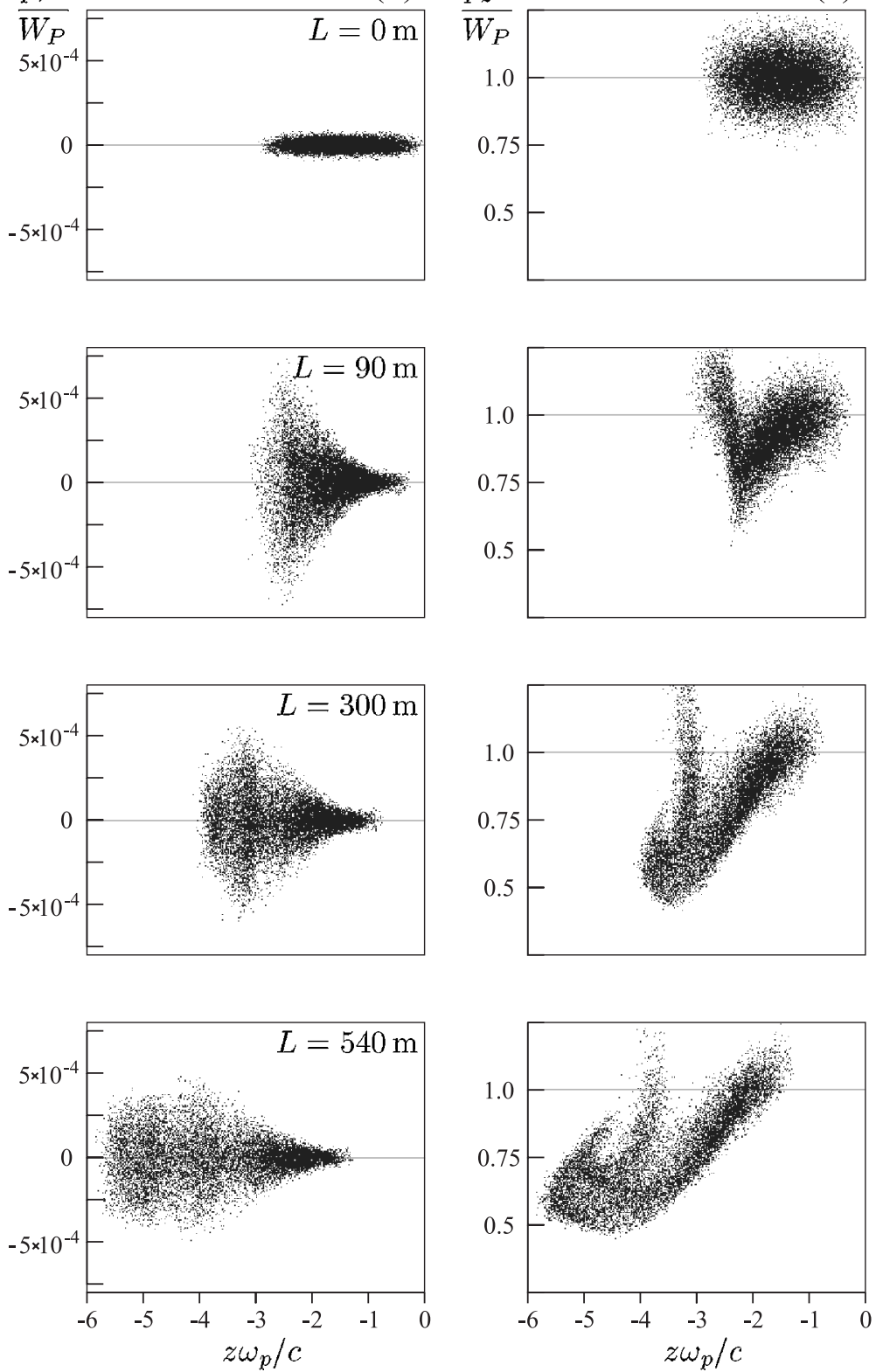

FIG. 3. (Color) Driver density distribution (a), radial momentum $p_{r}$ of driver protons versus longitudinal coordinate $z$ (b), and longitudinal phase portrait of the driver (c) at four propagation distances: $L=0 \mathrm{~m}, 90 \mathrm{~m}, 300 \mathrm{~m}$, and $540 \mathrm{~m}$. 


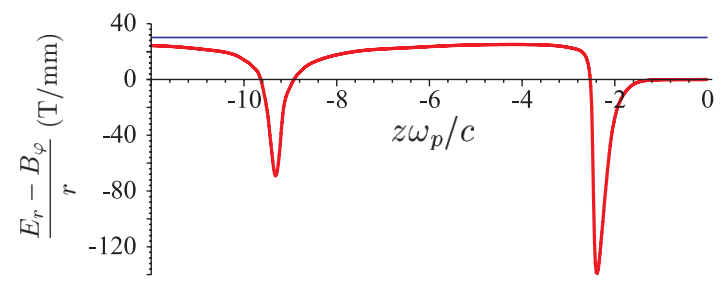

FIG. 4. (Color) Focusing strength of the plasma wave in the near-axis region. The horizontal line shows the focusing by a uniform ion column.

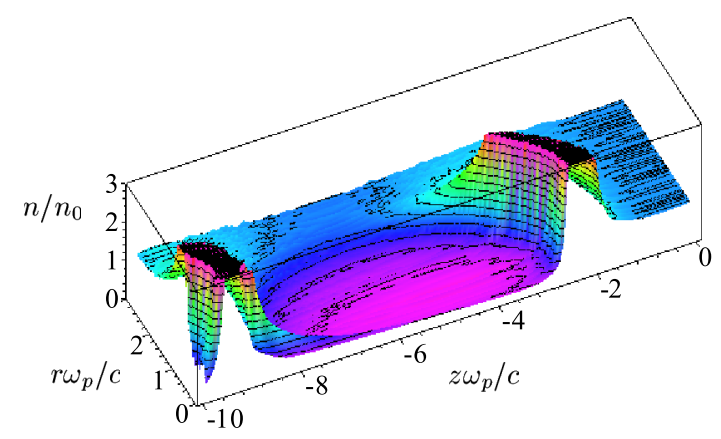

FIG. 5. (Color) The density of plasma electrons in the unloaded wakefield $(L=90 \mathrm{~m})$.

At optimum conditions, the plasma response to the proton beam (Fig. 5) is very similar to the blowout regime observed for dense electron drivers, with all good features of this regime present: almost linear focusing of accelerated electrons, longitudinal field independent on radial position, and high efficiency of energy takeoff. The unloaded electric field also has the saw-toothed profile [Fig. 6(a), red curve] typical to the blowout regime.

As the driver gets depleted, lower energy protons from its central part lag behind the others thus causing elongation of the driver (Fig. 3, $L=300 \mathrm{~m}$ and $L=540 \mathrm{~m}$ ). As a consequence, the wakefield amplitude [Fig. 6(c)] and the energy content of the wave get smaller; this is the main effect limiting the interaction distance and the energy of accelerated particles. The elongation length for proton drivers can be estimated in the same manner as formula (4) was obtained. Assuming the maximum decelerating field $\sim \alpha E_{0}$, we find the energy $W$ of slowest driver particles as a function of the traveled distance $L$ :

$$
W=W_{P}\left(1-L / L_{0}\right)
$$

and their velocity

$$
v \approx c\left(1-\frac{1}{2 \gamma_{0}^{2}\left(1-L / L_{0}\right)^{2}}\right) .
$$

The wakefield amplitude drops considerably when these particles shift backward by the distance $\sim c / \omega_{p}$ with respect to particles of the initial energy $W_{P}$ and velocity $v_{0}$, or if
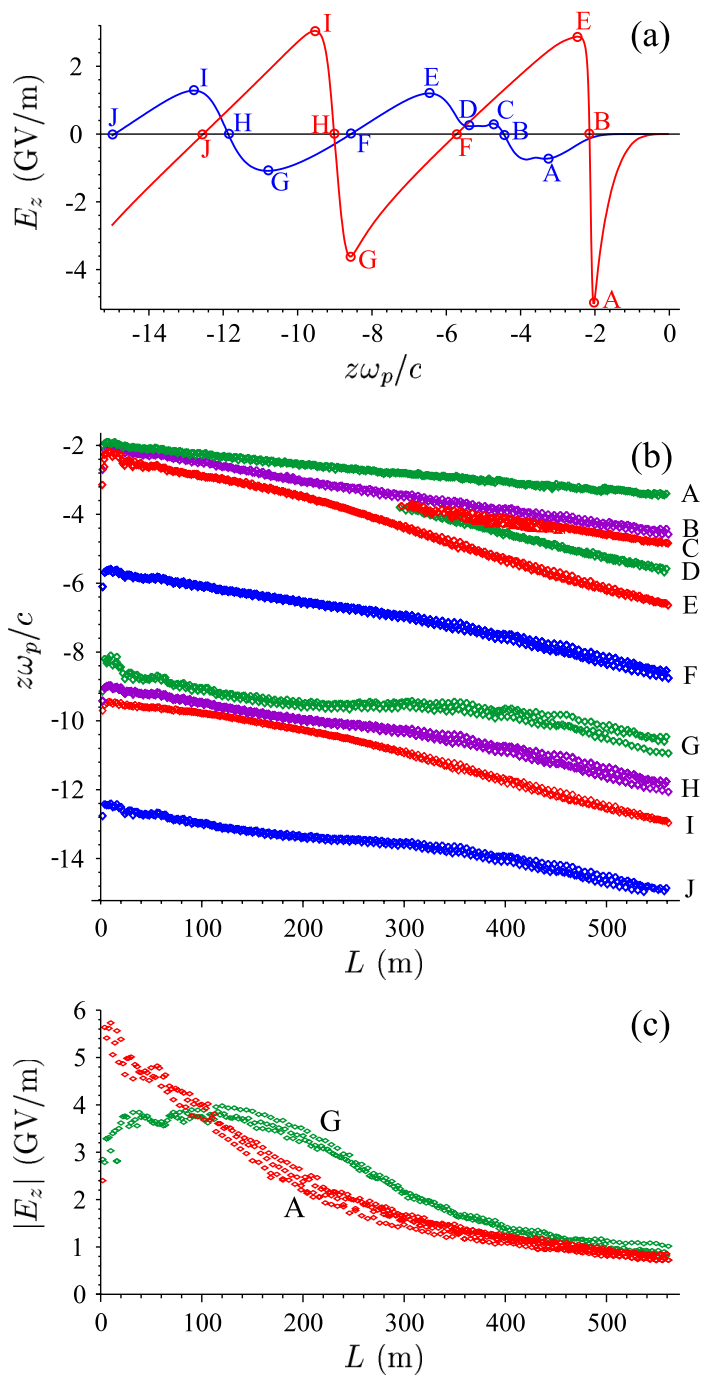

FIG. 6. (Color) (a) The unloaded on-axis electric field generated by a fresh driver $(L=30 \mathrm{~m}$, red) and by a depleted one $(L=$ $540 \mathrm{~m}$, blue); (b) locations of field extrema and zeros as functions of the traveled distance [letters correspond to the points marked in (a) by circles]; (c) field amplitude in the first two local minima. The plasma density here is constant: $n_{0}=10^{15} \mathrm{~cm}^{-3}$.

$$
\frac{c}{\omega_{p}} \sim \int_{0}^{L} \frac{\left(v_{0}-v\right)}{c} d L=\frac{L^{2}}{2 \gamma_{0}^{2}\left(L_{0}-L\right)} .
$$

This equality determines the acceleration distance $L$ and the maximum energy gain of witness particles $W_{e m}$, which is roughly $W_{P} L / L_{0}$, as functions of the dimensionless parameter

$$
A_{w}=\frac{2 \gamma_{0}^{2} c}{L_{0} \omega_{p}}=\frac{2 \gamma_{0} m \alpha}{m_{P}}
$$

through the relation

$$
A_{w}=\frac{x^{2}}{1-x}, \quad x=\frac{L}{L_{0}} .
$$




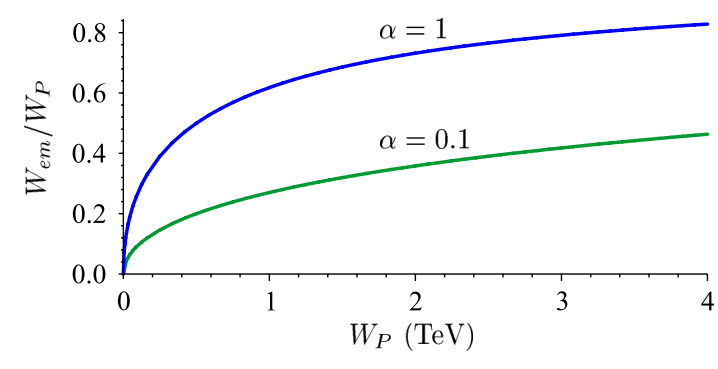

FIG. 7. (Color) Relative witness energy gain as a function of the driver energy for two dimensionless amplitudes of the plasma wave.

The dependence of the energy ratio $W_{e m} / W_{P}$ on the proton driver energy is illustrated in Fig. 7 for two values of the dimensionless amplitude $\alpha$.

There are two limiting cases of expression (16):

$$
\begin{gathered}
A_{w} \gg 1: L \approx L_{0}, \quad W_{e m} \approx W_{P} \\
A_{w} \ll 1: L \approx L_{0} \sqrt{A_{w}}, \quad W_{e m} \ll W_{P} .
\end{gathered}
$$

The latter one is obviously inefficient. For protons, the boundary between the limiting cases $\left(A_{w}=1\right)$ is at the energy $W_{P} \sim \alpha^{-1} \mathrm{TeV}$. Thus, proton driven plasma wakefield acceleration makes sense only at multi-TeV beam energies and in heavily loaded plasmas. For our reference case, $L \sim 200 \mathrm{~m}$ and $W_{e m} \sim 0.6 \mathrm{TeV}$ in reasonable agreement with simulations [Fig. 6(c)].

\section{OPTIMUM ACCELERATION}

If the plasma density is constant along the beam line, then points of field maximum are not stationary in the speed-of-light frame, which is undesirable for acceleration of the witness. To visualize the relative shifts of the wakefield, we plot in Fig. 6(b) the location of points at which the on-axis electric field $E_{z}$ is zero or reaches an extremum. The labeling of the points is shown in Fig. 6(a). There are two main effects responsible for observed shifts. First, the field pattern moves back as a whole due to a relatively low relativistic factor of the driver. Second, as the driver gets depleted, the field profile gradually changes from the sawtoothed to almost sinusoidal one [Fig. 6(a)] causing field minima to shift forward. For the place of presumable witness location (near the point " $G$ "), the two effects partly compensate each other, but still we need some tapering of the plasma density to keep the witness in optimum conditions along the whole acceleration distance.

To find the optimum tapering, we realize the following algorithm. We divide the whole interaction distance into sections of the length $L_{q}$. In each section, we adjust the plasma density so that to maximize the quantity

$$
\Delta W_{e}-10 \delta W_{e},
$$

where $\Delta W_{e}$ is the gain of witness energy, and $\delta W_{e}$ is change of witness energy spread in this section. The factor

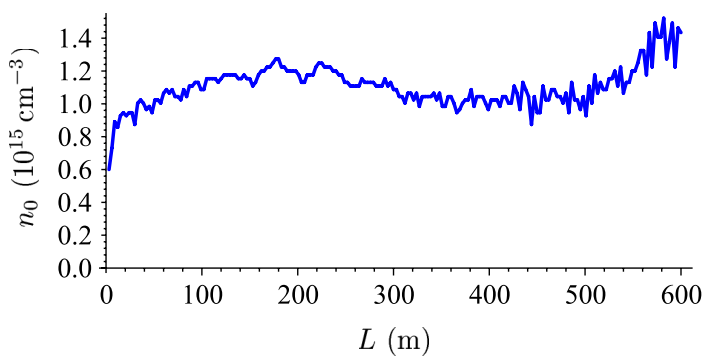

FIG. 8. (Color) Optimum dependence of the plasma density on the traveled distance.

of 10 in (19) is chosen somewhat arbitrary to emphasize the importance of a low energy spread. The average (over the whole interaction length) plasma density is controlled by the distance between the driver and the witness; the shorter
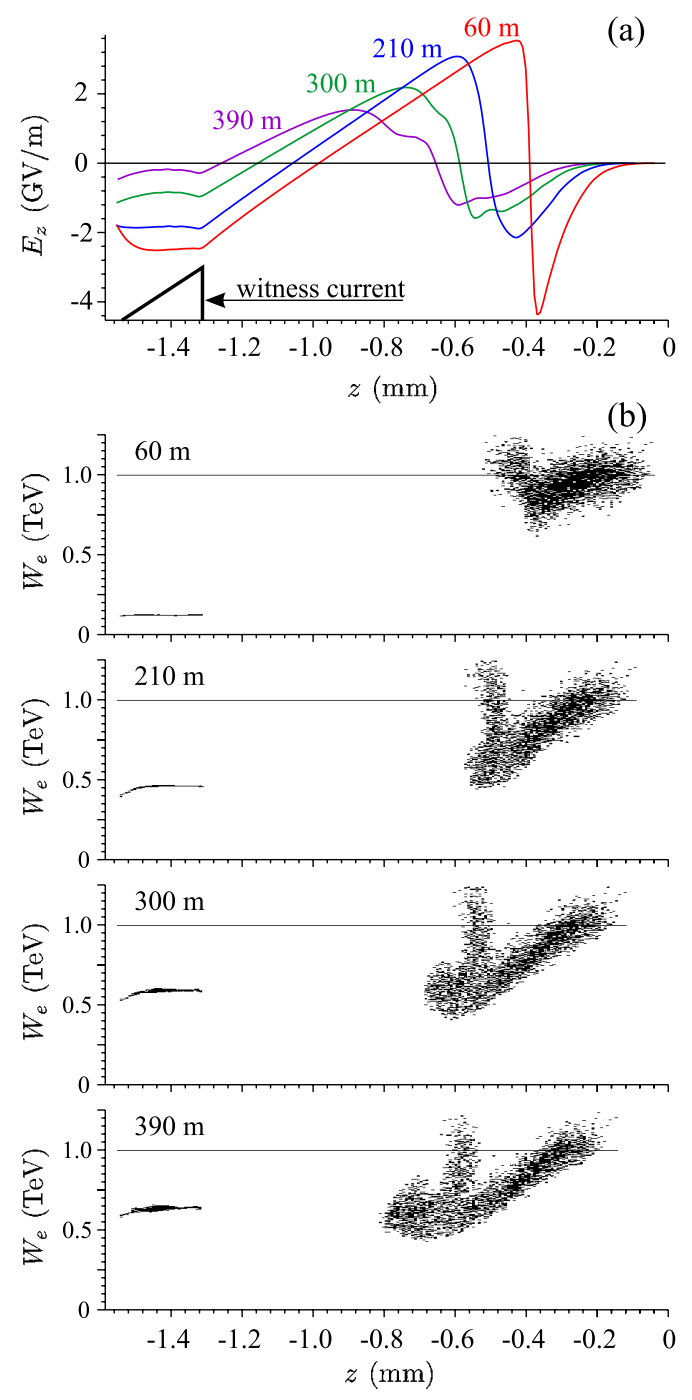

FIG. 9. (Color) On-axis electric field (a) and longitudinal phase portrait of beams (b) at several propagation distances. The black triangle shows charge distribution and location of the witness beam. 
the distance, the higher density maximizes the expression (19). The charge distribution in witness is triangular since this shape provides the minimum energy spread in both linear [19] and moderately pumped blowout regimes [14]. The witness length $(240 \mu \mathrm{m})$ is chosen empirically to minimize the overall energy spread.

The obtained density profile is shown in Fig. 8. Density variations within $\pm 40 \%$ from the average value are sufficient to keep the whole witness in an almost constant field [Fig. 9(a)]. As the driver elongates [Fig. 9(b)], the accelerating field decreases and eventually becomes zero, which means the energy content of the wave is too low to keep the whole witness of this charge in a constant accelerating field at any density of the plasma. By this moment, the witness beam is accelerated to $660 \mathrm{GeV}$ [Fig. 10(a)] in surprisingly good agreement with the estimate (16). The energy spread grows mainly at the final stage of acceleration [Fig. 10(b)] and can be kept low at some sacrifice in the energy gain. The wakefield of a depleted driver fails not only to accelerate the witness, but also to keep it tightly focused near the axis, so the witness is scattered transversely after some point marked by crosses in Fig. 10.

The result presented in Fig. 10 is better than those published in [11] due to twice higher plasma density and more careful optimization of other parameters.

As far as driver elongation sets the ultimate limit on the acceleration distance, the idea comes to mind to put the witness into the second or third accelerating bucket (i.e., far from the driver) and, when the driver becomes long, abruptly reduce the plasma density, so that the witness jumps one basket forward. One could expect that, in a lower density plasma, the wavelength is longer, and the long driver would still excite the wakefield, so acceleration
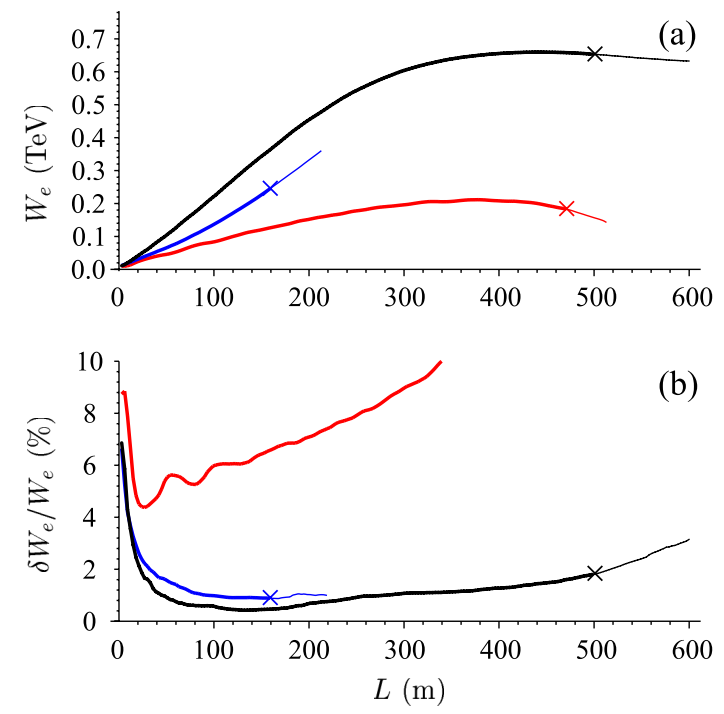

FIG. 10. (Color) Witness energy (a) and energy spread (b) versus traveled distance for optimum (black), low (blue), and high (red) driver emittances. The crosses mark points of witness depopulation. would continue (though at a lower rate). Practically, this method does not work since the angular spread of the driver is matched to the strong focusing force of the high-density plasma and turns out to be too large for the low-density plasma. As a consequence, the driver quickly scatters after the density steps down, so the acceleration distance cannot be increased by this trick.

\section{DEVIATIONS FROM THE OPTIMUM}

Unlike the electron-driven blowout regime, for proton beams the best wakefield structure is realized at some optimum values of initial driver radius and emittance. Higher values result in larger equilibrium radius of the driver, lower driver density, and lower energy content of the wave. At lower values and smaller equilibrium radius, the driver imparts so large transverse momentum to nearby plasma electrons that these electrons escape from the wave taking away a considerable amount of energy. The latter feature differentiates proton drivers from electron ones for which the radius, if it is smaller than the bubble radius, has little effect on the wakefield strength. Figures 10 and 11 illustrate these statements. The low-emittance case corresponds to $\epsilon_{P}=0.005 \mathrm{~mm} \mathrm{mrad}$ and initial radius $\sigma_{r}=$ $0.3 \mathrm{~mm}$; dense diverging jets of plasma electrons are clearly seen in this case [Fig. 11(b)], and the bubble is smaller than in the optimum regime [Fig. 11(a)]. In the high-emittance case, $\epsilon_{P}=0.02 \mathrm{mmmrad}$ and $\sigma_{r}=$ $0.6 \mathrm{~mm}$; the bubble disappears [Fig. 11(c)]. In both cases, the acceleration rate and witness energy gain are substantially lower than in the optimum regime (Fig. 10), so the optimum in initial driver radius is rather sharp.
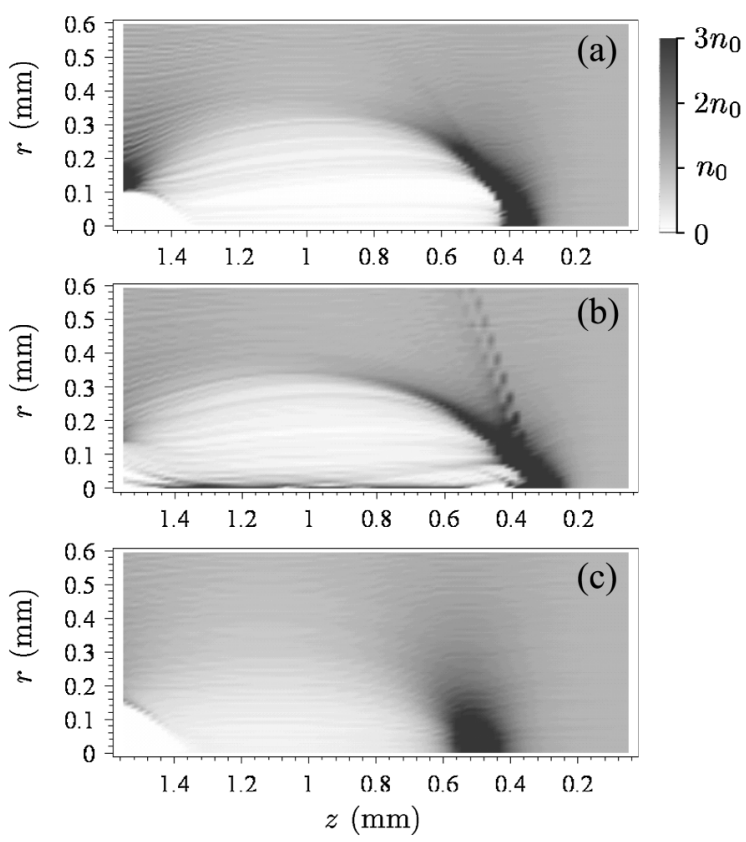

FIG. 11. Maps of the plasma electron density for optimum (a), low (b), and high (c) driver emittances at $L=60 \mathrm{~m}$. 


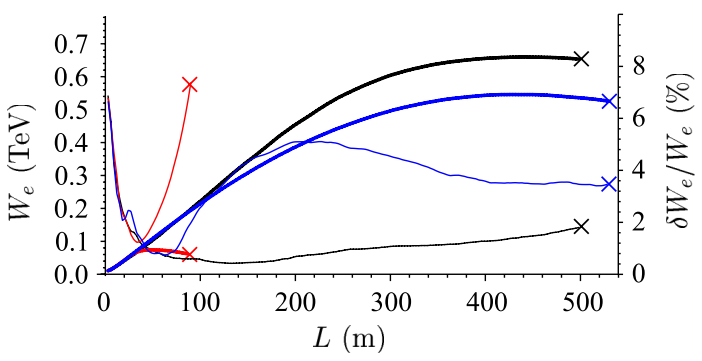

FIG. 12. (Color) Witness energy (thick lines) and energy spread (thin lines) versus traveled distance for the optimum acceleration (black), no density tapering and $n_{0}=10^{15} \mathrm{~cm}^{-3}$ (blue), and no quadrupole focusing (red).

The importance of density tapering and quadrupole focusing is illustrated by Fig. 12. With a constant plasma density, the witness energy gain is reduced insignificantly, while the energy spread blows up. With no external focusing, acceleration is not possible at all since the driver disperses at the first hundred meters.

For beam parameters from Table I, the optimum witness length $(240 \mu \mathrm{m})$ is about $1.5 c / \omega_{p}$. Deviations of any sign from this value result in increase of the energy spread (Fig. 13). The short witness also gains less energy due to shorter acceleration distance, since a weak wakefield of the depleted driver fails to accelerate too dense electron bunch.

The effect of the average plasma density on the witness energy (Fig. 14) is in line with results of Sec. III. Increasing

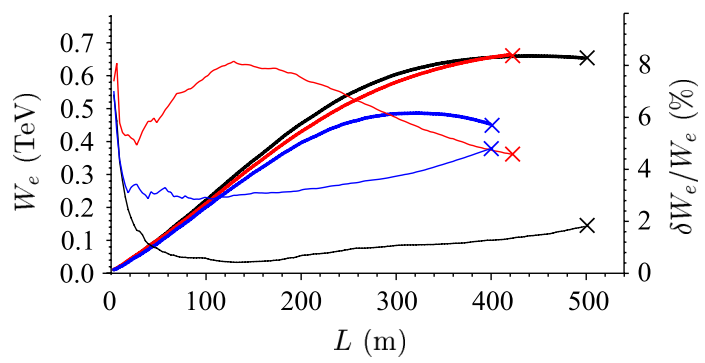

FIG. 13. (Color) Effect of the witness length: witness energy (thick lines) and energy spread (thin lines) versus traveled distance for witness lengths $0.16 \mathrm{~mm}$ (blue), $0.24 \mathrm{~mm}$ (black), and $0.35 \mathrm{~mm}$ (red).

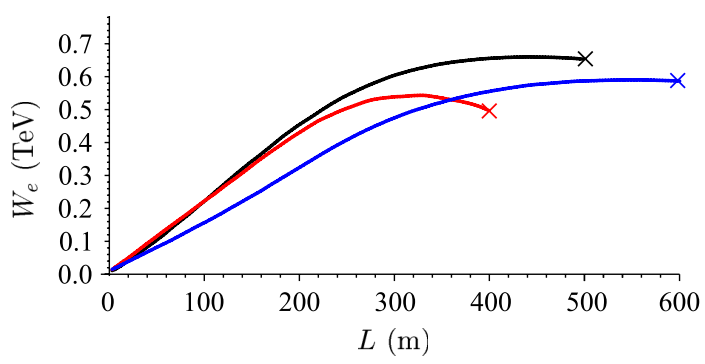

FIG. 14. (Color) Effect of the plasma density: witness energy growths for various average plasma densities: $10^{15} \mathrm{~cm}^{-3}$ (black), $4 \times 10^{14} \mathrm{~cm}^{-3}$ (blue), and $2 \times 10^{15} \mathrm{~cm}^{-3}$ (red).
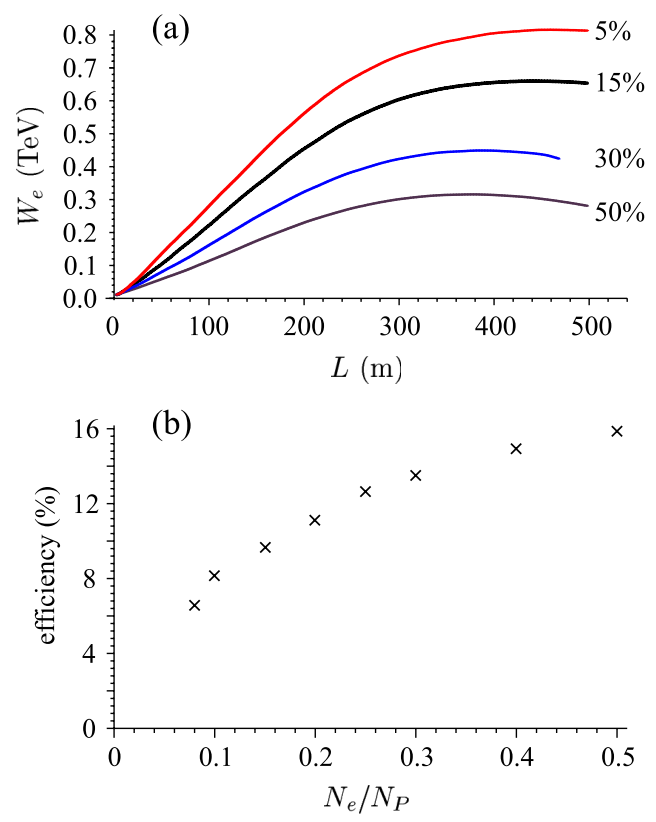

FIG. 15. (Color) Effect of the witness charge: (a) witness energy growth for various witness-to-driver charge ratios; (b) efficiency of driver-to-witness energy transfer as a function of witness charge.

the plasma density with respect to the optimum value does not result in an increase of the accelerating field, but reduces the energy content of the wave and the final witness energy. Decreasing the density mainly reduces the acceleration rate; the energy gain is reduced to a smaller extent.

Curves of energy gain for various witness charges are shown in Fig. 15(a). For each curve, the witness length was adjusted to minimize the energy spread and maximize the peak energy. As expected, higher charges result in lower acceleration rates and final energies. The acceleration distance also reduces with the increase of the witness charge. The overall efficiency of driver-to-witness energy transfer increases with the witness charge, but remains at the 10\%-15\% level [Fig. 15(b)].

\section{SUMMARY}

Let us emphasize main findings of the paper.

If compressed longitudinally to the submillimeter scale, proton beams can be used as drivers for plasma wakefield acceleration, but only beams of very high energies (well beyond $1 \mathrm{TeV}$ ) and fully loaded plasmas can provide an energy gain of accelerated electrons as high as the driver energy. The proton beam must be focused by quadrupole magnets surrounding the plasma column to prevent the beam head from fast emittance-driven erosion.

For efficient excitation of the wakefield, the beam must have a submillimeter length. The length of the beam controls the wakefield amplitude, while the beam population is responsible for energy content of the excited wave. 
Because of the "flow-in" effect, the plasma density providing the highest wakefield amplitude is almost an order of magnitude lower than those determined by the linear wakefield theory.

The drive beam in the plasma quickly comes to a transverse equilibrium, which is characterized by density peaking near the axis and emittance variation along the beam in accordance with the focusing strength of the wakefield.

At optimum conditions, the plasma response to the proton driver is very similar to the blowout regime.

The effect limiting the acceleration distance and thus the energy gain of the witness is that of driver elongation due to unequal energy depletion of different driver parts. The lag of the driver as a whole with respect to the light speed, however, does not lead to any serious limitation and can be completely compensated by a proper plasma density tapering along the interaction channel.

There is an optimum driver radius providing the highest wakefield. At a smaller radius, a considerable part of driver energy is spent at wave breaking. A larger radius results in weaker fields just because of a lower driver density.

With the triangular-shaped density distribution of the witness and proper choice of the witness length and location, it is possible to keep the witness energy spread below $1 \%$. For higher witness charges, the acceleration rate is lower, while the efficiency of driver-to-witness energy transfer is higher. For the $1 \mathrm{TeV}$ proton driver [11], typical parameters of the optimized plasma wakefield accelerator are energy gain $660 \mathrm{GeV}$, acceleration distance $400 \mathrm{~m}$, and beam-to-beam efficiency $10 \%$.

\section{ACKNOWLEDGMENTS}

The author greatly appreciates stimulating discussions with A. Caldwell, A. Pukhov, G. Xia, and A. Terekhov. Various parts of this work are supported by Russian Science Support Foundation, RF President's Grants
No. MD-2995.2009.2 and No. NSh-7792.2010.2, Russian Ministry of Education Grant No. RNP-2.1.1/3983, and RFBR Grants No. 09-02-00594 and No. 08-01-00622.

[1] E. Esarey, C. B. Schroeder, and W. P. Leemans, Rev. Mod. Phys. 81, 1229 (2009).

[2] C. Joshi, Phys. Plasmas 14, 055501 (2007).

[3] T. Katsouleas, Phys. Plasmas 13, 055503 (2006).

[4] E. Esarey, P. Sprangle, J. Krall, and A. Ting, IEEE Trans. Plasma Sci. 24, 252 (1996).

[5] T. Tajima and J.M. Dawson, Phys. Rev. Lett. 43, 267 (1979).

[6] P. Chen, J. M. Dawson, R. W. Huff, and T. Katsouleas, Phys. Rev. Lett. 54, 693 (1985); 55, 1537 (1985).

[7] W. P. Leemans et al., Nature Phys. 2, 696 (2006).

[8] I. Blumenfeld et al., Nature (London) 445, 741 (2007).

[9] R. D. Ruth, A. W. Chao, P.L. Morton, and P. B. Wilson, Part. Accel. 17, 171 (1985).

[10] A. M. Kudryavtsev, K. V. Lotov, and A. N. Skrinsky, Nucl. Instrum. Methods Phys. Res., Sect. A 410, 388 (1998).

[11] A. Caldwell, K. Lotov, A. Pukhov, and F. Simon, Nature Phys. 5, 363 (2009).

[12] S. Lee, T. Katsouleas, R. G. Hemkel, and W. B. Mori, Phys. Rev. E 64, 045501 (2001).

[13] J. B. Rosenzweig, B. Breizman, T. Katsouleas, and J. J. Su, Phys. Rev. A 44, R6189 (1991).

[14] K. V. Lotov, Phys. Plasmas 12, 053105 (2005).

[15] K. V. Lotov, Phys. Plasmas 5, 785 (1998).

[16] K. V. Lotov, Phys. Rev. ST Accel. Beams 6, 061301 (2003).

[17] T. Eichner et al., Phys. Rev. ST Accel. Beams 10, 082401 (2007).

[18] W. Lu, C. Huang, M. M. Zhou, W.B. Mori, and T. Katsouleas, Phys. Plasmas 12, 063101 (2005).

[19] T. Katsouleas, S. Wilks, P. Chen, J. M. Dawson, and J. J. Su, Part. Accel. 22, 81 (1987). 